around the edge of the tracheal wound, which evidently were held down while the tube was in position. Upon the removal of the tube they formed an obstruction to the passuge of air through the larynx as soon as the tracheal wound contracted. Dr. Jacobi, in a paper on "The Pathology and Treatment of Group," published in the American Journal of Obstetrics, May, 1868, called attention to the same condition interfering with the removal of the tube in several patients apon whom he had operated for tracheotomy. $\mathrm{Mr}$. Thomas smith, in a paper on the "Obstacles to the reestablishment of Natural Respiration after Performance of Tracheotomy," published in the Medico-Chirurgical Transactions, vol. xlviii., also relates several cases in which the presence of these granulations prevented the removal of the tube. In Dr. Jacobi's cases, and in my own, a fenestrated tube was used, and I infer from the description given by Mr. Smith that these tubes were used in his cases. In my second case, where a closed cannula was used, and allowed to remain in four weeks, no granulations were seen, though carefully looked for. In my third case, where the tube remained in eleven months, a non-fenestrated tube was also used, and there would probably have been no difficulty in its removal, after a few weeks, if other troubles had not intervened. These cases seem to prove that the fenestra which is found in nearly all the tracheotomy tubes made by American instrument makers, and which is for the purpose of testing the ability of the patient to breathe through the larynx when the tube is closed, is not only useless but injurious, probably causing by its irritation the growth of these granulations. Dr. Lewis A. Sayre reported a case before the Medical Society of the State of New York, in 1864, in which fatal hremorrhage took place, on the seventh day, after the operation in consequence of a slough through a large vein in the neighbourhood of the wound, caused by the pressure of the edges of the fenestra of the tube. In the paper already referred to by $\mathrm{Mr}$. Thomas Smith a letter from Trousseau is published, in which he says, "I advise you whenever you can to leave a closed cannula in the trachea until the child has breathed for ten days in a natural manner. The cannula, with a dorsal opening, is a aseless and often dangerous instrument, for, if you compare the calibre of the cannula with that of the trachea, you will see that the section of the trachea is more than double the area of that of the cannula. The air, therefore, can pass freely between them. On the other hand, the tissues project through the dorsal opening of the cannula, and thereby the inflammation is kept up." In my second case, after the granulations had disappeared, the closed cannula was no hindrance to the child's breathing in a natural manner. His voice was as loud and distinct before the final removal of the tube as after ; in fact, I could perceive no difference whatever. One other point in connexion with this case, which I did not refer to in the history, is this : the first tube which was used had an immovable collar. The nonfenestrated tube had the usual movable collar, which, allowing a considerable degree of motion, prevented a certain amount of irritation during swallowing, coughing, and other movements of the patient's neck. In regard to the size of the tube, Mr. Robert M. Parker, in a paper on Tracheotomy in Membranous Laryngitis, published in the Medico-Cairurgical Transactions, vol. lxii., advises the use of the largest tube that can be got into the trachea without actual violence, for the reasons that it will afford the patient a better supply of air, and also allow the tracheal secretions to be removed with greater facility, which feature in the after-treatment of these cases he considers of paramount importance. He also advises the movable collar and the beveling of the extremity of the tube, and recommends that the tube be as short as is consistent with safety; he finally proposes a cannula in which the curve resembles the Gothic rather than the Roman arch. If a tightly fitting tube such as he advises be used, it should of necessity have a fenestra, as the air could not pass between it and the walls of the trachea. In the other cases detailed, the advice given by Trousseau was strictly followed, and the tube was not removed until the voice was clear and the child could breathe freely through the mouth. I think the size of the tube should always be smaller than the calibre of the trachea. It should have a movable collar, and the lower extremity should be beveled from before downwards and backwards. I consider that the shorter the tube the better. In order to make these tubes as light as possible, I have had them manufactured for me by Mr. Tiemann, of New York, of aluminium. This does not tarnish nor corrode like silver. The shape of the tube is here represented. I have never used the Durham tube, which has the advantage of being shortened at will. The excellent tube recommended by Mr. Bryant has this same advantage, com. bined with a ball-and-socket movement, which imparts.

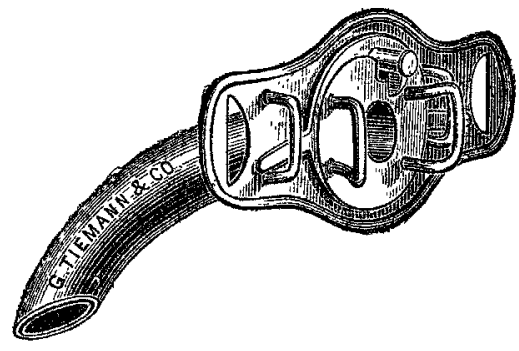

mobility to the tube when in the trachea. This tube could be improved, I think, by being made of aluminium.

The conclusions that I would draw from these cases are-

1. That the tube used should be somewhat smaller than the calibre of the trachea, should have no dorsal opening. and should be made with a movable collar or ball-and-socket attachment.

2. No attempt should be made to remove the tube permanently until the patient is able to breathe freely for some days through the mouth with the tube closed.

3. That granulations around the margin of the tracheal wound will be less likely to occur if no source of irritation exist in connexion with the tube.

\section{FOUR YEARS' TREATMENT OF INSANITY AT GARLAND'S ASYLUM, WITH REMARKS.}

\section{BY J. A. CAMPBELL, M.D., F.R.S.E. (Concluded from p. 498.)}

HAVING disposed of the general results and shown, so far as recovery, death, or asylum retention is concerned, the disposal of these four years' admissions (it seems to me unnecessary to deal with a small proportion, chiefly imbeciles, removed to workhouses or to friends), I shall now shortly discuss what was done in the way of treatment, dealing first with medicinal treatment.

Table of Summary of Treatment.

\begin{tabular}{c|c|c|c|c}
\hline Years. & $\begin{array}{c}\text { No. of } \\
\text { admissions. }\end{array}$ & \multicolumn{3}{|c}{ Treated by } \\
\hline 1879 & 112 & 50 & 20 & 2 \\
1880 & 129 & 47 & 11 & 4 \\
1881 & 114 & 39 & 8 & 1 \\
1882 & 140 & 53 & 3 & 2 \\
\hline
\end{tabular}

The tonic and blood restorer that I have principally used has been iron and quinine, the tincture of the muriate in from five to ten minim doses, and quinine in one grain doses; in a few cases the citrate of iron and quinine was used, in several Easton's syrup, also tineture of the muriate and tincture of quassia, and in one or two instances a solution of strychnia and quinine. I have also used very freely malt extract and cod-liver oil in the treatment of these cases, though I do not include either in the above table. Malt extract has wonderful weight-producing effects, and is of use also in persistent refusal of food; it seems to assist digestion in unsalivated and unmasticated food. In a case that I fed for a long time with the tube during 1881 and 1882, while I gave malt extract with feeding, the patient gained in weight, when it stopped he lost. I also used stimulants freely in the recent cases wherever I thought them likely to be of use. Bitter beer (Bass), London stout (Barclay and Perkins), Islay whisky (Lagavulin distillery), were the forms used. I have already discussed the mode in which stimulants are made 
use of in this asylum, so do not enter on the subject here. ${ }^{1}$ The treatment by hypnotics here merely signifies that for usually a limited number of nights sleep was artificially produced, and in all but two cases chloral was the drug used; in two bromide of potassium, a very mild sedative, was given. In the nine cases that were treated by sedatives continuously during the day for some time, bromide of potassium was the agent used in all but one case, and in that case a mixture containing tincture of opium and tincture of hyoscyamus was used; this latter was a general paralytic. In a considerable number of cases, where syphilitic taint was known to exist or suspected, iodide of potassium was used in combination with tincture of cinchona; in several cases marked mental amelioration rapidly ensued.

It is unnecessary to say that the functional ailments of the female patients were specially treated, and also other general or local physical disease in patients of either sex. All the epileptics who were admitted were treated continuously with bromide of potassium ; in fact, for many years all the epileptics in the asylum who would take medicine have been continuously on this treatment with good effect, and their state is regularly examined into at short intervals. I have used blisters to the head in five cases, and continuous counterirritation by tincture of iodine in one case, though in none of these cases has recovery taken place, yet in two it may; they were all cases threatening to become totally demented. I should like to hear the experience of others as to blisters in such cases, I have used the morning cold shower bath freely and with good effect in a large number of cases during the summer months, but I have nothing to add to what I have already said ${ }^{2}$ on the value of this as a curative agent in certain given forms of insanity. I may shortly state that during these four years I have found it unnecessary to use continuous sedative treatment, or sleep-producing draughts, to the same extent that I did during the previous five years. I believe this to be due to the greater attention devoted to the recent cases, the more extended open-air treatment in force, and the greater absence of asylum-made excitement which now exists here. Nothing can be more prejudicial to new patients than to be herded among an unruly lot of noisy chronic lunatics, and $I$ am also of opinion that the plan of having very large wards to contain over fifty and up to a hundred patients is inimical to proper care, comfort, and safety for the patient as well as detrimental to cure. Attendants in a very large ward so subdivide care, responsibility, and attention, that when anything happens it is quite impossible to find who is to blame, and when it is the work of many little real interest or attention is paid to any one patient. Better results are certainly arrived at by subdivision of both patients and attendants.

Attendance.-Hitherto too much has been expected from asylum attendants; most asylums have too few attendants for really proper curative treatment. Each new patient should, if at all excitable, have a special attendant for some time after admission. The increase in night attendants which has taken place in English asylums, owing to the repeated suggestions of the Commissioners in Lunacy, is a much-needed step in the right direction. The residuum of the last ten years' admissions into this asylum is a much more easily dealt with, more orderly, cleaner, and less noisy lot, than the older residua, and this $I$ believe is entirely owing to the greater care and attention both by day and night that was expended on them, especially during the first portion of their residence here.

Out-door exercise. - I dealt at length in my former paper with this subject. I need only say that weather, and the patients' state allowing of it, I have kept all the excited patients out all day with excellent results; the excitement quickly subsides in most cases, the general health improves, and natural sleep becomes re-established; but whlle this treatment is most useful in the majority of excited cases, there are some in which it may do harm.

Rest in bed.-In going over some asylums, I have heard it pointed out as a thing to be proud of how few cases there were in bed. This is a wrong state of matters if bed would have been the best place for some of the patients. There are certain cases that, instead of being walked out, really require rest in bed and constant and careful feeding to quiet them - the excitement, the result of nervous ex-

1 Note on the absence of beer in an Asylum dietary, THE LanceT May 14th, 1881.

2 The Shower Bath in Insanity, Journal of Mental Science, Jan. 1873. haustion in aged and weak individuals. In many cases of epilepsy in excitable patients, if the patient were put to bed before coming out of a fit and left quiet, an attack of excitement would be warded off.

Seclusion and restraint. - I find that I have used seclusion in cases of violent paroxysms of excitement, when I feared injury to or from the patient, to the following extent (this excludes cases of epilepsy and epileptical excitement): in 1879 , ten individuals on eleven occasions ; in 1880, six individuals on seven occasions; and in 1882, nine individuals on nine occasions. This record applies in this particular to the whole asylum population, averaging 450 , not alone to the admissions during the four years. I am quite aware that in some asylum reports much is made of seclusion never being used; but if the patients are like those who come under my observation, I am very sure that in certain cases it is the safe, the humane, and the proper treatment. I have used restraint in all surgical cases where absolute rest was required. I do not think that the healing of a wound or broken bone should be delayed or imperilled because at one time restraint was abused.

What is the value of a recovery? Irrespective of the gain to the individual of his sanity and also of the amount which his labour may bring him during his future life, the relief to the union of his settlement in this county, at the present asylum rate of $9 s$. $7 \frac{1}{2} d$. a week, with the death-rate for twenty-one years averaging $7 \cdot 7$ per cent., means thirteen years' cost, or $£ 325$, if he keeps out for life. I have always thought this aspect of a recovery was not brought into proper prominence : outlay in sufficient medical attendance is more protitable than in stone and lime for additions, or in addi. tional officers for the purposes of management.

Are the recoveries real? Who can say till their life history is finished? It would be as wrong to detain in an asylum after a reasonable probation a person who as far as can be judged appears sane, as to keep in a hospital a patient re. covered from pneumonia for fear exposure should bring on another attack. In some cases I am sure rapid discharge is the proper course for the patient's sake, in others a lengthened period of probation. To form any opinion as regards the real value of discharges in a short period would be fallacious, but the results of ten years' admissions, discharges, readmissions, and deaths will allow of discussion. In the ten years ending 1882,1182 patients were admitted; 268 were readmissions, the recovery rate was $47 \cdot 3$, the average death-rate 8.1 , and the increase by retention was 121 . The 560 patients who recovered consisted of 508 individuals ; 52 appeared more than once-viz., 1 five times, 2 four times, 10 three times, and 39 twice; 138 of those recovered were resident under three months. Of this number 28 have so far been readmitted, but 4 only within the year of discharge.

My experience convinces me that, given the same character of admissions, the more individual medical care, attention, consideration, and judicious curative treatment an insane patient receives, the greater will be his chance of recovery, the higher will be the asylum recovery rate. We must in this branch of medicine plod along like the rest of our medical brethren, adding by persistent observation to our know. ledge, though it may be very slowly and little by little.

I am glad to say that in this asylum the percentage of recoveries has so far not lessened, as shown by the concluding table :-

Table showing General Results of Three Periods comprising Fourteen. Years.

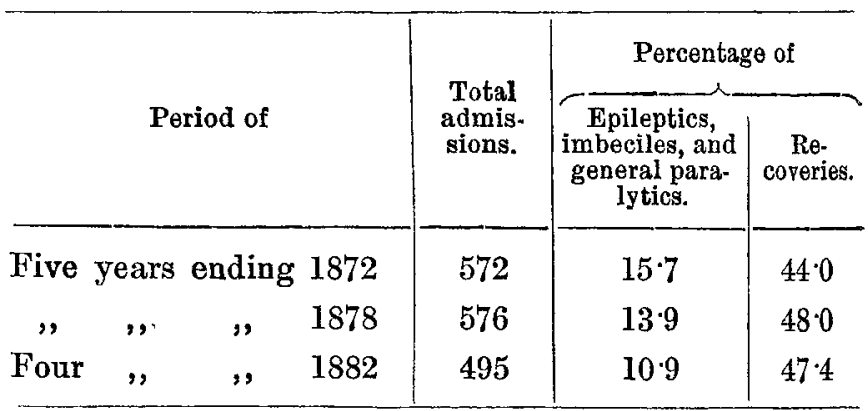

In the last period ten chronic patients who had been boarded in another asylum owing to overcrowding here were brought back ; they count in the admissions, and reduce the recovery rate, which is calculated on the admissions. Were it not for this the recovery rate would stand at $48^{\circ} 4$, the highest of the three periods given. 